\title{
Methods to Measure the Rotational Inertia of Rocket Projectiles of Ultra Large Diameter
}

\author{
Yu Yang ${ }^{1, a}$, Bing Wang ${ }^{2}$ \\ ${ }^{1}$ Changchun University of Science and Technology,Changchun,Jilin province,China \\ ${ }^{2}$ Guangdong Ningyuan Science Park Development Company Limited \\ ayangyu1983@sina.com
}

\begin{abstract}
Key words: Rotational Inertia;Torsion Method;Combined Spring
Abstract.There are lots of methods used to measure the rotational inertia; however, very limited measures can be adopted to measure in a high precision manner the rotational inertia of rocket projectiles of ultra large diameter. Based on this situation, the paper has put forward a method called Torsion Method to measure the rotational inertia of rocket projectiles of ultra large diameter and made an analysis on precision of the method. In addition, a deep analysis is conducted aiming at the combined type of torsion springs that are serving as the kernel technology of the method.
\end{abstract}

\section{Introduction}

Moment of inertia,the physical quantity representing the size of inertia of rotational objects, is an important engineering technical parameter. Therefore, when it comes to issues about rotational dynamics, measurement of moment of inertia will be involved.

In national defense industry, it is needed to measure the moment of inertia of all various kinds of guided missiles including rocket projectiles, nuclear warheads, in order to determine the initial disturbance and flight stability. So, it has important practical significance to measure the moment of inertia .

Large rocket, a production of modern war and high technology, has been widely applied in military field. As it is a vital factor which is not only related to the national security but also represents the comprehensive strength of a country, it is very important to improve the reliability and accurate strike ability of the weapons system. As moment of inertia has influences on both initial disturbance and flight stability, measuring the moment of inertia can not only provide important basis for the research and design, calculation of flight speed and acceleration, interpretation of the launching results, calculation of the unbalance of rocket projectile, but also information for the optimization of shape and flying attitude. However, in the process of measuring the moment of inertia, a larger mass of the tested object will bring a lower accuracy of the moment of inertia. As a result, measuring the moment of inertia of large rocket has become a technical difficulty in measuring the moment of inertia. It has irreplaceable practical significance for improving China's technology in the field to analyze and study the method and technique of measuring the moment of inertia of current large rockets.

\section{Principle of Measurement Involved in the Torsion Method}

The rotational inertia of rocket projectiles of ultra large diameter is measured with a view to offering static parameters necessary for the trajectory calculation and then providing important basis for the assessment of product performance. The Torsion Method is presented in this paper to measure the rotational inertia of rocket projectiles of ultra large diameter and profound analysis is made of this method.

There are many methods that can be used to measure the rotational inertia, including such commonly used ones as Compound Pendulum Method, Falling Body Method, Bifilar Pendulum Method and Three-wire Pendulum Method; however, a certain problems are existed in all the methods mentioned above. In recent years, the Rotation Method starts to be adopted both at home and abroad to measure the rotational inertia of rocket projectiles of ultra large diameter. The principle of measurement involved in the method is shown in Figure1. 


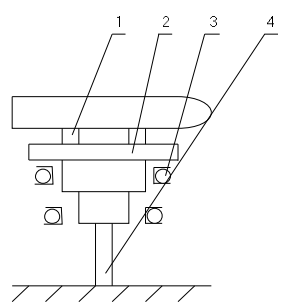

Figure1Principle of measuring rotation inertia

1 V type rack; 2 Platform; 3 Bearing; 4 Torsion bar

The pendular equation of pendular object is expressed as:

$$
\frac{d^{2} \theta}{d t^{2}}+2 \alpha \frac{d \theta}{d t}+\frac{k \theta}{J}=0
$$

In this equation, $J$ represents the rotational inertia of pendular object; $a=c / 2 J$; $c$ represents the damping coefficient of air; $\theta$ represents the rotation angle; $k$ represents a constant.

$$
\omega^{2}=\frac{k}{J}-\frac{c^{2}}{2 J}
$$

In this equation, $\omega$ represents the angular frequency of natural vibration, in which $\omega=2 \pi / T$; under normal circumstances, the damping coefficient of air is very little, namely $c<<$; therefore, $c^{2} / 2 J \rightarrow 0$, then $J=\frac{k}{4 \pi^{2}} T^{2}$, Suppose $A=\frac{k}{4 \pi^{2}}$, then :

$$
J=A T^{2}
$$

Because the projectile must be charged in a pendular object for pendulum, therefore:

$$
J_{d}+J_{0}=A T^{2}
$$

In this equation, $J_{d}$ represents the rotational inertia of projectile; $J_{0}$ presents the rotational inertia of the pendular object itself; $A$ represents the constant related to the structure of pendular object; $T_{0}$ represents the period of pendulum required for the joint pendulum of projectile and pendular object.

With a view to determining the value of $J_{0}$, it is a must to measure the value of $T_{0}$, which represents the period of pendulum when the pendular object is in idle swing, first:

$$
J_{0}=A T_{0}^{2}
$$

Then measure the value of $T_{c}$, which represents the period of period of pendulum required for the joint pendulum of standard sample piece and pendular object:

$$
\begin{aligned}
& J_{s}+J_{0}=A T_{s}^{2} \\
& J_{d}=J_{s}\left|\frac{T_{c}^{2}-T_{0}^{2}}{T_{s}^{2}-T_{0}^{2}}\right|
\end{aligned}
$$

In the equation, $J_{s}$ represents the rotational inertia of standard sample piece and can be calculated; therefore, the value of $J_{d}$ can be calculated based on the measured values of $T_{c}, T_{0}$, $T_{s}$ and the known value of $J_{s}$.

\section{Precision Analysis}

The torsion bar is adopted in this apparatus to offer the restoring moment, which is vastly superior to springs from the perspective of stress produced, because smaller damping can be achieved by use of torsion bar.

Although different apparatus is adopted for the polar moment of inertia and transverse moment of inertia, the equation of motion can be concluded as follows:

$$
J \phi^{\prime \prime}+K \phi=0
$$

Based on this simple harmonic motion, the equation used to measure the moment of inertia can 
be obtained as follows:

$$
J=A T^{2}-J_{0}
$$

In this equation, $A$ and $J_{0}$ can be measured by the standard sample piece directly before the measurement is conducted. During measurement, only the period of torsional vibration of the object under test placed on the apparatus is required to be measured and then the moment of inertia of object under test can be calculated.

That the errors are mainly derived from that measurement of the vibration period $T$. However, the errors produced in $T$ are mainly from the two aspects as follows: one is the error occurring during measurement; the other is errors produced due to the neglect of damping moment.

The errors occurring during measurement can be neglected because it's very easy to ensure the precision of measurement.

According to the vibration theory, the damping effect on the apparatus mentioned above is taken into consideration and only the period is lengthened. Suppose $T^{\prime}$ represents the period in which the damping effect is considered:

$$
T^{\prime 2}=T^{2}\left[1+\left(\frac{0.25 \beta}{\pi}\right)^{2}\right]
$$

In the equation above, $\beta$ represents logarithmic decrement and its value depends on the times of torsional vibration; suppose the times of torsional vibration is $5-6$, then $\beta=0.25$ and the error is about $0.04 \%$. The times of actual vibration will be much more than that determined here. It can be seen that the apparatus can meet requirement of measurement accuracy being smaller than $1 \%$.

\section{Mechanical Analysis of Combined Type of Spring}

The combined type of spring serves as the core technology used to measure the moment of inertia; the maximum torque it can provide has determined the quality of rocket projectiles of large diameter measured.

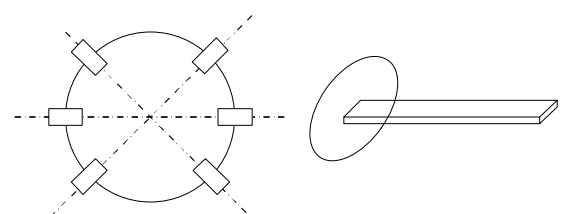

Figure2 Combined torsion spring

As shown in Figure 2 that the flange plates are selected for 2 end faces; the cross section of strip is rectangular shaped; $n$ pieces of strips are evenly distributed on the circumference with a radius of $R$; the cross section of strip is $h \times b$; the length of strip is $l$; suppose the elastic modulus of its material is $E$. Suppose the force required to produce the unit displacement on the head of strip 3 is $P_{1}$, then:

$$
P_{1}=\frac{3 E I}{l^{3}}
$$

In which, $I$ represents the moment of inertia of the strip, $I=\frac{b h^{3}}{12}$.

Then, the torsional rigidity of the whole torsion spring $K$ can be expressed as follows:

$$
K=\frac{n E b h^{3} R^{2}}{4 l^{3}}
$$

Considering that the main failure mode of torsion springs after being loaded axially is the loss of stability, the axial load can be expressed as follows:

$$
P=\frac{\pi^{2} n E b h^{3}}{48 l^{2}}
$$

The maximum permissible stress on any one point of strip can be expressed as follows: 
$\sigma_{\text {max }}=\frac{M_{\max }}{\frac{b h^{2}}{6}} \leq[\sigma]$

Then, the maximum torque that can be offered by a strip is expressed as follows:

$M_{\max }=\frac{b h^{2}}{6}[\sigma]$

Therefore, the following equation can be obtained:

$P_{1}=\frac{b h^{2}[\sigma]}{6 l}$

The maximum torque that can be offered by the combined type of torsion spring $\mathrm{M}$ is expressed as follows:

$$
M=\frac{n R b h^{2}[\sigma]}{6 l}
$$

\section{Conclusions}

It is shown from the mathematical analysis made of the methods to measure the moment of inertia of rocket projectile of ultra large diameter that the relative measurement accuracy of the method is less than $0.1 \%$ and its error is less than $5 \%$; therefore, the method can fully meet the requirement of detection on the moment of inertia of the rocket projectile of ultra large diameter in China.

\section{References}

[1]Li Huayi, Zhang Yingchun, Li baohua. Design of large bullet rotary inertia instrument.J. Weapon automation. 22(2003)4-6

[2] Yu Zhihui. Measurement of rotational inertia of small missiles. J.Tactical missile technology. 2(2002)38-46

[3] Li Huayi,Zhang Yingchun, Li Baohua. Analysis and Design of the Moment of lnertia Measuring Instrument with High Accuracy. J.ACTA METROLOGICA SINICA.25(2004)250-253

[4] Qu Xiuquan,Jiao Yinghou,Chen Zhaobo.A calculation method of equivalent moment of inertia for one-DOF planar linkage.J.JOURNAL OF HARBIN INSTITUTE OF TECHNOLOGY. 36(2004)620-623

[5] Li Tonghua,Zhu Zhangang,Shang Chunmin.Error Analysis of Measuring Bullet Rotary Inertia with Torsional Vibration Method. J. Journal of Changchun University of Science and Technology. 30(2007)63-65

[6] Liu Wei,Zhang Yang,Ma Xin.Measurement method for moment of inertia based on binocular vision.J.Chinese Journal of Scientific Instrument.35(2014)1972-1978 